\title{
A Comparative Matched Study of Breast-Conserving Therapy and Modified Radical Mastectomy in Iranian Women
}

\author{
Mohammad Houshyari ${ }^{1}$, Afshin Rakhsha ${ }^{1}$, Mohammadreza Khademi ${ }^{2}$ and Amir Shahram Yousefi \\ Kashi (iD ${ }^{1, *}$ \\ ${ }^{1}$ Cancer Research Center, Faculty of Medicine, Shahid Beheshti Univeristy of Medical Sciences, Tehran, Iran \\ ${ }^{2}$ Student Research Committee, Department of Radiation Oncology, School of Medicine, Shahid Beheshti Univeristy of Medical Sciences, Tehran, Iran \\ "Corresponding author: Cancer Research Center, Faculty of Medicine, Shahid Beheshti Univeristy of Medical Sciences, Tehran, Iran. Email: shahpoo2002@yahoo.com
}

Received 2019 May 02; Revised 2019 May 22; Accepted 2019 July 20.

\begin{abstract}
Background: Breast cancer is the most frequent diagnosed visceral cancer with 13400 new cases annually among Iranian women. Modified radical mastectomy (MRM) was the old standard surgical treatment in patients with breast cancer from 70 years ago and breast-conserving surgery (BCS) with adjuvant radiotherapy was the standard treatment from 30 years ago.

Objectives: In this matched historical cohort study, we compared two types of therapy for breast carcinoma: breast-conserving therapy (BCT), and MRM.

Methods: Medical records of 6000 patients with breast cancer from the database of Cancer Research Center of Shahid Beheshti University of Medical Sciences were reviewed and 1310 patients who had been treated by BCT (group A) or MRM (group B) were selected based on a time- stratified 1:1 between September 2002 and December 2014 as 10 baseline variables. By log-rank test analysis, their local recurrence (LR), disease free survival (DFS) and overall survival (OS) were evaluated and compared.

Results: In groups A and B, the median age at the diagnosis time was 48.11 and 48.64 years old, respectively. Median follow-up time was 71 months since April 2015. 1.68\% of patients in group A and 1.07\% of patients in group B had local recurrence during the five years of follow up, $(\mathrm{P}=0.173)$. In the group $\mathrm{A}$ and $\mathrm{B}$, five years DFS were $87.94 \%$ and $80.46 \%,(\mathrm{P}<0.001)$ and five years $\mathrm{OS}$ were $89.31 \%$ and $83.02 \%$, respectively $(\mathrm{P}=0.041)$.

Conclusions: The findings of this study showed that 5-year DFS and 5-year OS in BCT group were better than in the MRM group. Longer follow-up time of the patients to compare 10-year DFS and 10-year OS or even 20-year OS between two groups is recommended.
\end{abstract}

Keywords: Breast Cancer, Breast-Conserving Therapy, Modified Radical Mastectomy, Survival

\section{Background}

Breast cancer is the most frequent diagnosed visceral cancer and is the second cause of death due to cancer among Iranian women based on the cancer registry system (1).

There are 13400 new cases of breast cancer with an incidence rate of 32 in 100000 in Iranian women annually. In Iran, breast cancer is diagnosed 10 years earlier than most developed countries, therefore, we had major health and treatment problems of burden of this disease (2).

For more than 70 years, radical mastectomy and modified radical mastectomy (MRM) were known as a standard surgical treatment in the breast cancer. After that, breast conserving surgery (BCS) with adjuvant radiotherapy was developed as the standard treatment in early-stage breast cancer from 30 years ago.

There are seven prospective clinical randomized trials that have shown that breast-conserving therapy (BCT) is equivalent to modified radical mastectomy (MRM) in overall survival, in spite of higher rate of local recurrence (LR) (3-9).

\section{Objectives}

In this historical cohort study, we compared local recurrence, distant recurrence, 5-year DFS, and 5-year OS in patients with breast cancer who were treated by BCT or MRM at Cancer Research Center of the Shahid Beheshti University of Medical Sciences between September 2002 and December 2014.

\section{Methods}

In this historical cohort study, a consecutive series of 6000 patients with breast cancer were treated by BCT or 
MRM. Data were extracted from the database of Cancer Research Center, Shahid Beheshti University of Medical Sciences, a referral breast clinic in Tehran, Iran. Our sampling method was a time-stratified 1:1 approach for selecting 1310 women who were eligible for our study. BCS was followed by adjuvant radiotherapy to the whole breast with or without boost was done in 655 cases and MRM with or without adjuvant radiotherapy was determined in 655 cases between September 2002 and December 2014.

All 1310 patients were matched based on a timestratified 1:1 approach with stage I to III without distant metastasis and were matched based on 10 baseline variables (age at diagnosis, stage of tumor, tumor histology, tumor grade, number of positive nodes, pathologic tumor size, type of chemotherapy ER, PR and HER2 status) that are believed for having a significant role with distant recurrence, LR, DFS and OS, based on previous studies. The exclusion criteria were: patients with breast cancer who did not have acceptable follow up after initial diagnosis, patients with metastatic, and patients without pathologic diagnosis. The inclusion criteria were: patients with breast cancer with stage I to III who had acceptable follow up after initial diagnosis and had all 10 baseline variables (age at diagnosis, stage of tumor, tumor histology, tumor grade, number of positive nodes, pathologic tumor size, type of chemotherapy ER, PR and HER2 status).

All patients with breast cancer had pathologic diagnosis which was performed by biopsy or surgery of the primary breast tumor. In the BCT group, resection of the primary tumor with an adequate negative margin of axillary lymph node dissection (ALND) or sentinel lymph node biopsy (SLNB) was performed and all patients were followed by adjuvant radiotherapy to the whole breast with or without boost to the primary site of tumor. In the MRM group, the surgical procedure included resection of the whole breast with an adequate surgical negative margin with ALND were followed by adjuvant radiotherapy to the chest wall with or without irradiation to the regional lymph nodes.

After all treatments were over, every breast cancer patient was visited and examined every 3 to 6 months for five years and yearly afterward. The patients underwent mammography annually. Breast cancer patients who did not have acceptable follow up after initial diagnosis were excluded from the study. In the case of clinical symptoms or signs of any recurrences, patients underwent imaging or biopsy to identify any recurrences.

Until April 2015, breast cancer patients had a follow-up period of 41 months. As the time interval between initial diagnosis and local or distant recurrence was defined as DFS and the time interval between initial diagnosis and death was defined as OS.
In the act provided by Shahid Beheshti University of Medical Sciences, the ethical regulations dictated were approved to review of the medical records for the purposes of our study (ethical code: IR.SBMU.MSP.REC.1396.358).

Differences in all variables on local and distant recurrence and death were evaluated and analyzed by the chisquare test. DFS and OS rates were estimated by KaplanMeier analysis and compared by the Log-rank test. P value $<0.05$ was considered significant. All statistical analyses were performed using the IBM SPSS 22.0.

\section{Results}

In BCT group and MRM group, the median age was 48.11 years (range 22 - 84 years) and 48.64 years (range 17 - 85 years), respectively. In the both groups 68 patients $(10.4 \%)$ had age $\leq 35$ years and 587 patients (89.6\%) had age $>35$ years.

Total of 563 patients (86\%) had pathologic report of infiltrating ductal carcinoma, 79 patients (12\%) had other pathology except infiltrating ductal carcinoma and 13 patients (2\%) had unknown pathology reports in both groups. Fifty-two cases (8\%) had well-differentiated grade, 354 patients (54\%) had moderately differentiated grade, 184 patients $(28 \%)$ had poorly differentiated grade, and in both groups the grade of 65 cases (10\%) was unknown.

In group A and group B there were 197 patients (30\%) with zero involved lymph nodes, 268 patients (41\%) with $\leq$ 3 pathologic positive lymph nodes, 144 patients (22\%) with $\geq 4$ pathologic positive lymph nodes, and 46 patients (7\%) with unknown pathologic lymph nodes.

In group A and group B there were 105 cases (16\%) with tumor size $>5$ centimeters $(\mathrm{cm}), 452$ cases $(69 \%)$ with tumor size $\leq 5 \mathrm{~cm}$, and 98 cases (15\%) with unknown tumor size.

Based on tumor stage, in group A and B, 59 cases (9\%) had stage I, 321 cases (49\%) had stage II, 216 cases (33\%) had stage III, and 59 patients (9\%) were with unknown stage.

In group A and B, 219 patients (33\%) received NAC and 437 patients $(67 \%)$ received AC.

For ER status in group A and group B there were 426 (65\%) patients with ER positive, 203 cases (31\%) with ER negative and 26 (4\%) cases were with unknown ER receptor status.

For PR status in group A and group B there were 360 (55\%), 236 (36\%), and 59 (9\%) patients with PR positive, PR negative, and unknown PR receptor status, respectively.

In BCT group and MRM group there were 151 (23\%), 439 (67\%), and 65 patients (10\%) with HER2 positive, HER2 negative, and unknown HER2 status, respectively. 
Table 1 summarizes baseline characteristics and the clinical-pathological features of 1310 adult patients with breast cancer (655 patients in each group).

\begin{tabular}{|c|c|c|}
\hline Characteristics & Group A: BCT (N=655) & $\begin{array}{c}\text { Group B: MRM }(\mathrm{N}= \\
655)\end{array}$ \\
\hline \multicolumn{3}{|l|}{ Age, $y$} \\
\hline Median (range) & $48.11(22-84)$ & $48.64(17-85)$ \\
\hline$\leq 35$ & $68(10.4)$ & $68(10.4)$ \\
\hline$>35$ & $587(89.6)$ & $587(89.6)$ \\
\hline \multicolumn{3}{|l|}{ Tumor histology } \\
\hline IDC & $563(86)$ & $563(86)$ \\
\hline Others & $79(12)$ & $79(12)$ \\
\hline Unknown & $13(2)$ & $13(2)$ \\
\hline \multicolumn{3}{|l|}{ Chemotherapy type } \\
\hline NAC & $219(33)$ & $219(33)$ \\
\hline AC & $437(67$ & $437(67)$ \\
\hline \multicolumn{3}{|l|}{ Tumor size, cm } \\
\hline$\leq 5$ & $452(69)$ & $452(69)$ \\
\hline$>5$ & $105(16)$ & $105(16)$ \\
\hline Unknown & $98(15)$ & $98(15)$ \\
\hline \multicolumn{3}{|l|}{ Nodal status } \\
\hline Node-negative & $197(30)$ & $197(30)$ \\
\hline $\begin{array}{l}1-3 \text { positive } \\
\text { nodes }\end{array}$ & $268(41)$ & $268(41)$ \\
\hline $\begin{array}{l}\geq 4 \text { positive } \\
\text { nodes }\end{array}$ & $144(22)$ & $144(22)$ \\
\hline Unknown & $46(7)$ & $46(7)$ \\
\hline \multicolumn{3}{|l|}{ Tumor stage } \\
\hline I & $59(9)$ & $59(9)$ \\
\hline II & $321(49)$ & $321(49)$ \\
\hline III & $216(33)$ & $216(33)$ \\
\hline Unknown & $59(9)$ & $59(9)$ \\
\hline \multicolumn{3}{|l|}{ Tumor grade } \\
\hline $\begin{array}{l}\text { Well } \\
\text { differentiated }\end{array}$ & $52(8)$ & $52(8)$ \\
\hline $\begin{array}{l}\text { Moderately } \\
\text { differentiated }\end{array}$ & $354(54)$ & $354(54)$ \\
\hline $\begin{array}{l}\text { Poorly } \\
\text { differentiated }\end{array}$ & $184(28)$ & $184(28)$ \\
\hline Unknown & $65(10)$ & $65(10)$ \\
\hline \multicolumn{3}{|l|}{ Receptor status } \\
\hline ER positive & $426(65)$ & $426(65)$ \\
\hline ER negative & $203(31)$ & $203(31)$ \\
\hline Unknown & $26(4)$ & $26(4)$ \\
\hline PR positive & $360(55)$ & $360(55)$ \\
\hline PR negative & $236(36)$ & $236(36)$ \\
\hline Unknown & $59(9)$ & $59(9)$ \\
\hline HER2 positive & $151(23)$ & $151(23)$ \\
\hline HER2 negative & $439(67)$ & $439(67)$ \\
\hline Unknown & $65(10)$ & $65(10)$ \\
\hline
\end{tabular}

Abbreviations: AC, adjuvant chemotherapy; BCT, breast-conserving therapy; MRM, modified radical mastectomy; NAC, neo-adjuvant chemotherapy

${ }^{a}$ Values are expressed as No. (\%) unless otherwise indicated.
Since December 2014, a median follow-up time was 71 months (range 48 - 192 months), 11 cases (1.68\%) of local recurrence were shown in the BCT group and seven patients (1.07\%) of local recurrence were shown in MRM group during the five years of follow up. Thus the 5-year local recurrence- free survivals were $98.32 \%$ in BCT group and 98.93\% in MRM group. There was not any significant local recurrence differences between BCT group and MRM group based on the log-rank test 5-year local recurrence analysis, $(P=0.173, R R=1.13,95 \% C I=1.05-1.22)$ (Table 2 ). Then patients in BCT group did not show more local recurrence than MRM group.

Table 2. Comparison of Local Recurrence and Distant Recurrence During 5-Years, Between Group A and Group B.

\begin{tabular}{|ccc}
\hline & Group A & Group B \\
\hline Included patients, $\mathbf{N}$ & 655 & 655 \\
\hline Local recurrence during 5-years, No. (\%) & $11(1.68)$ & $7(1.07)$ \\
\hline RR 95\%CI & $1.13(1.05-1.22)$ \\
\hline P value (log-rank test) & 0.173 \\
\hline $\begin{array}{l}\text { 5-years local recurrence-free survival, No. } \\
\text { (\%) }\end{array}$ & $644(98.32)$ & $648(98.93)$ \\
\hline RR 95\%CI & $1.13(1.05-1.22)$ \\
\hline P value (log-rank test) & \multicolumn{2}{c}{0.173} \\
\hline Distant recurrence during 5-years, No. (\%) & $68(10.38)$ & $121(18.47)$ \\
\hline RR 95\%CI & $1.78(1.58-1.98)$ \\
\hline P value (log-rank test) & $<0.001$ \\
\hline 5-years distant disease-free survival, No. (\%) & $587(89.62)$ & $534(81.53)$ \\
\hline RR 95\%CI & $1.78(1.58-1.98)$ \\
\hline P value (log-rank test) & $<0.001$ \\
\hline
\end{tabular}

Abbreviations: $\mathrm{CI}$, confidence interval; RR, relative risk

In BCT group, 68 patients (10.38\%) were diagnosed with distant metastasis during of the 5-year follow up and 121 patients (18.47\%) had distant recurrence in MRM group. Thus the 5-year- distant recurrence free survival were $89.62 \%$ in BCT group and $81.53 \%$ in MRM group. We found a significant difference between two groups as distant recurrence free survival based on log-rank test analysis $(\mathrm{P}<0.001, \mathrm{RR}$ $=1.78,95 \% \mathrm{CI}=1.58-1.98$ ) (Table 3 ).

The five-year DFS rate was $87.94 \%$ and $80.46 \%$ in BCT and MRM groups, respectively. We observed a significant difference between two groups as the five-year DFS based on the log-rank test analysis $(\mathrm{P}<0.001)$ (Table 3). Then patients in BCT group showed better DFS than MRM group.

In BCT and MRM groups, the five-year OS rate was 89.31\% and $83.02 \%$, respectively. We showed a significant difference between two groups as the five-year OS based on logrank test analysis $(\mathrm{P}=0.041)$ (Table 3$)$. Then patients in BCT 
Table 3. Comparison of the Five Years Disease Free Survival (DFS) Rate and Five Years Overall Survival Rate (OS), Between Patients with Breast Cancer in Group A and Group B

\begin{tabular}{|ccc}
\hline & Group A & Group B \\
\hline Included patients, $\mathbf{N}$ & 655 & 655 \\
5-year DFS Rate, \% & 87.94 & 80.46 \\
P value (log-rank test) & \multicolumn{2}{c}{$<0.001$} \\
5-year OS Rate, \% & 89.31 & 83.02 \\
\hline P value (log-rank test) & & 0.041 \\
\hline
\end{tabular}

Abbreviations: DFS, disease free survival; OS, overall survival

group showed better OS than MRM group.

\section{Discussion}

The results of this study showed that 5-year DFS and 5-year OS rates in BCT group were better than the MRM group. In the current study, which has been conducted at Cancer Research Center of the Shahid Beheshti University of Medical Sciences, we compared local recurrence, distant recurrence, 5-year DFS, and 5-year OS in 1310 matched patients who underwent MRM or BCT. To the best of our knowledge, it is the greatest series in Iran.

There are seven prospective randomized clinical trials which have found that BCT is equivalent to MRM as OS in breast cancer patients, in spite of a higher rate of LR (39). In many Western countries, BCT has been performed in clinical practice for more than 25 years. In Iran, there are lower rates of BCT in comparison to western countries that might relate to some factors such as socioeconomic factors and concern over the increased risk of LR. In addition, distant recurrence appears to have been related to both breast cancer patients and their physicians.

We minimized the selection bias between BCT and MRM groups and all data were matched and analyzed according to 10 baseline variables (age at diagnosis, stage of tumor, tumor histology, tumor grade, number of positive nodes, pathologic tumor size, type of chemotherapy, ER, PR and HER2 status) that are believed for having a significant role with distant recurrence, LR, DFS, and OS based on previous studies.

In the BCT group and MRM group, the median age was 48.11 and 48.64 years old, respectively. In both groups, 68 cases $(10.4 \%)$ had age $\leq 35$ years and 587 cases $(89.6 \%)$ had age $>35$ years, and there were no differences in the frequency of patients $\leq 35$ years. These findings indicate that age is not an important factor to predict LR and distant metastases in both groups. This result is consistent with most studies $(8,9)$.
Wang et al. $(10,11)$ showed the 6-year LR-free survival (LRFS) rates was $98.2 \%$ in the BCT group and $98.7 \%$ in the MRM group $(\mathrm{P}=0.182)$. These findings were consistent with our study which showed that the 5-year LRFS was $98.32 \%$ in the BCT group and 98.93\% in the MRM group $(\mathrm{P}=0.173)$. They also showed the 6-year DFS rates in BCT and MRM groups was $91.3 \%$ and $86.3 \%(\mathrm{P}<0.001)$ that were consistent with our study, which found that the 5-year DFS rates were $87.94 \%$ in the BCT group and $80.46 \%$ in the MRM group $(\mathrm{P}<0.001)$. They could not show any comparison as overall survival between two groups, but in this study, we showed that the 5 -year OS was $89.31 \%$ in group A and $83.02 \%$ in group $\mathrm{B}(\mathrm{P}=0.041)$.

Veronesi et al. (12) found higher risk of LR, distant recurrence, and breast cancer mortality associated with BCS than MRM. These findings were inconsistent with our study which showed that BCT group did not have more local recurrence, distant recurrence, and breast cancer mortality than the MRM group.

Hwang et al. (13) demonstrated that in stage I and II of breast cancer, the 5-year PFS and the 5-year OS were worse in the MRM group than BCT group. These findings were consistent with this study, which found that the 5-year PFS and the 5-year OS in the BCT group were better than MRM group. In this study we compared stage I, II, and III and found the same results.

Yuan et al. (14) showed that BCT patients had worse LR , inferior 5-year DFS, and inferior 5-year OS than MRM cases as stage I, II, and III of breast cancer; in the present study, We showed 5-year DFS and 5-year OS in both groups were compatible, that were inconsistent with Yuan et al.'s study.

In another study carried out by van Dongen et al. (15) in July of 2000 showed the 10-year local recurrence rates were $20 \%$ in the BCT patients and $12 \%$ in the MRM cases (P $=0.01$ ). These findings were inconsistent with our study which that found the 5-year LR rates were $1.68 \%$ in the BCT cases and $1.07 \%$ in the MRM patients $(\mathrm{P}=0.173)$. They also showed that the 10-year OS rates in BCT and MRM groups were $66 \%$ and $65 \%(P=0.011)$, respectively. These findings were consistent with our study. Based on this study the 5year OS rates were $89.31 \%$ in group A and $83.02 \%$ in group $B$ $(\mathrm{P}<0.001)$.

Agarwal et al. (16) demonstrated the 5-year OS of BCT cases, a mastectomy alone cases, or a mastectomy with adjuvant radiation therapy were $97 \%$, $94 \%$, and $90 \%$, respectively $(\mathrm{P}<0.001)$. These findings were consistent with our study that the 5-year DFS was $87.94 \%$ in the BCT cases and $80.46 \%$ in the MRM cases $(\mathrm{P}<0.001)$, and the 5-year OS was $89.31 \%$ in the BCT patients and $83.02 \%$ in the MRM cases $(\mathrm{P}=0.041)$.They also found the 10 -year breast cancerspecific survival rates in the BCT group, in mastectomy alone group, and in mastectomy with radiation therapy 
group were $94 \%, 90 \%$, and $83 \%$, respectively ( $\mathrm{P}<0.001)$. In the present study, there were not any findings about 10year PFS and 10-year OS.

The limitations of this study included the missing data of some patients' information and the short follow up period.

\subsection{Conclusions}

The findings of this study showed that 5-year DFS and 5 -year OS rates in BCT group were better than the MRM group. The local recurrence rate in the BCT group was more than MRM group, but increased LR was not associated with worse DFS and OS. However, because of short follow up period, this study cannot prove that BCT is definitely superior to MRM.

\section{Acknowledgments}

This article has been extracted from the thesis written by Mr Mohammadreza Khademi in School of Medicine, Shahid Beheshti University of Medical Sciences (Registration No.: M162). We would also like to thank the staff of the Cancer Research Center, Shahid Beheshti University of Medical Sciences, for their contribution to maintenance of the patient records and data collection without whom, carrying out this project would be impossible.

\section{Footnotes}

Conflict of Interests: There is no conflict of interest to be declared.

Ethical Approval: In the act provided by Shahid Beheshti University of Medical Sciences, the ethical regulations dictated were approved to review of the medical records for the purposes of our study (ethical code: IR.SBMU.MSP.REC.1396.358).

Funding/Support: The authors received no financial support for the research, authorship, and/or publication of this article.

\section{References}

1. World Health Organization. The top 10 causes of death in 2014, fact sheet of WHO report. World Health Organization; 2008.

2. Goya M. Iranian Annual Cancer Registration Report 2005/2006. Ministry of Health and Medical Education, Health Deputy. Center for Disease Control and Prevention; 2007.

3. Atkins H, Hayward JL, Klugman DJ, Wayte AB. Treatment of early breast cancer: A report after ten years of a clinical trial. $\mathrm{Br}$ Med J. 1972;2(5811):423-9. doi: 10.1136/bmj.2.5811.423. [PubMed: 4624222] [PubMed Central: PMC1788035].
4. Gasparini G, Panizzoni GA, Dal Fior S, Germani B, Dall'Antonia F, Segato G, et al. Conservative surgery and irradiation (QUART) in the treatment of 243 stage I-II breast cancer patients. Anticancer Res. 1991;11(4):1635-40. [PubMed: 1746920].

5. Fisher B, Jeong JH, Anderson S, Bryant J, Fisher ER, Wolmark N. Twentyfive-year follow-up of a randomized trial comparing radical mastectomy, total mastectomy, and total mastectomy followed by irradiation. N Engl J Med. 2002;347(8):567-75. doi: 10.1056/NEJMoa020128. [PubMed: 12192016].

6. Jacobson JA, Danforth DN, Cowan KH, d'Angelo T, Steinberg SM, Pierce L, et al. Ten-year results of a comparison of conservation with mastectomy in the treatment of stage I and II breast cancer. N Engl J Med. 1995;332(14):907-11. doi: 10.1056/NEJM199504063321402. [PubMed: 7877647].

7. Hejazi E, Nasrollahzadeh J, Fatemi R, Barzegar-Yar Mohamadi L, Saliminejad K, Amiri Z, et al. Effects of combined soy isoflavone extract and docetaxel treatment on murine 4T1 breast tumor model. Avicenna J Med Biotechnol. 2015;7(1):16-21. [PubMed: 25926948]. [PubMed Central: PMC4388885].

8. Blichert-Toft M, Rose C, Andersen JA, Overgaard M, Axelsson CK, Andersen KW, et al. Danish randomized trial comparing breast conservation therapy with mastectomy: Six years of life-table analysis. Danish Breast Cancer Cooperative Group. J Natl Cancer Inst Monogr. 1992;(11):19-25. [PubMed: 1627427].

9. Sarrazin D, Le MG, Arriagada R, Contesso G, Fontaine F, Spielmann $\mathrm{M}$, et al. Ten-year results of a randomized trial comparing a conservative treatment to mastectomy in early breast cancer. Radiother Oncol. 1989;14(3):177-84. doi: 10.1016/0167-8140(89)90165-5. [PubMed: 2652199].

10. Yousefi Kashi AS, Yazdanfar S, Akbari ME, Rakhsha A. Triple negative breast cancer in Iranian women: Clinical profile and survival study. Int J Cancer Manage. 2017;10(8). doi: 10.5812/ijcm.10471.

11. Wang L, Ouyang T, Wang T, Xie Y, Fan Z, Lin B, et al. Breastconserving therapy and modified radical mastectomy for primary breast carcinoma: A matched comparative study. Chin J Cancer Res. 2015;27(6):545-52. doi: 10.3978/j.issn.1000-9604.2015.11.02. [PubMed: 26752928]. [PubMed Central: PMC4697104].

12. Veronesi U, Cascinelli N, Mariani L, Greco M, Saccozzi R, Luini A, et al. Twenty-year follow-up of a randomized study comparing breastconserving surgery with radical mastectomy for early breast cancer. N Engl J Med. 2002;347(16):1227-32. doi: 10.1056/NEJMoa020989. [PubMed: 12393819].

13. Hwang ES, Lichtensztajn DY, Gomez SL, Fowble B, Clarke CA. Survival after lumpectomy and mastectomy for early stage invasive breast cancer: The effect of age and hormone receptor status. Cancer. 2013;119(7):1402-11. doi: 10.1002/cncr.27795. [PubMed: 23359049]. [PubMed Central: PMC3604076].

14. Yousefi Kashi AS, Mofid B, Mirzaei HR, Azadeh P. Overall survival and related prognostic factors in metastatic brain tumors treated with whole brain radiation therapy. Res J Med Sci. 2010;4(3):213-6. doi: 10.3923/rjmsci.2010.213.216.

15. van Dongen JA, Voogd AC, Fentiman IS, Legrand C, Sylvester RJ, Tong $\mathrm{D}$, et al. Long-term results of a randomized trial comparing breastconserving therapy with mastectomy: European Organization for Research and Treatment of Cancer 10801 trial. J Natl Cancer Inst. 2000;92(14):1143-50. doi:10.1093/jnci/92.14.1143. [PubMed: 10904087].

16. Agarwal S, Pappas L, Neumayer L, Kokeny K, Agarwal J. Effect of breast conservation therapy vs. mastectomy on disease-specific survival for early-stage breast cancer. JAMA Surg. 2014;149(3):267-74. doi: 10.1001/jamasurg.2013.3049. [PubMed: 24429935]. 